\title{
On X-ray Variability in Seyfert Galaxies
}

\author{
T.J.Turner ${ }^{1,2}$, I.M. George ${ }^{1,3}$, K. Nandra ${ }^{1,3}$ D. Turcan ${ }^{4}$
}

\begin{abstract}
This paper presents a quantification of the X-ray variability amplitude for 79 ASCA observations of 36 Seyfert 1 galaxies. We find that consideration of sources with the narrowest permitted lines in the optical band introduces scatter into the established correlation between X-ray variability and nuclear luminosity. Consideration of the X-ray spectral index and variability properties together shows distinct groupings in parameter space for broad and narrow-line Seyfert 1 galaxies, confirming previous studies. A strong correlation is found between hard X-ray variability and $\mathrm{FWHM} \mathrm{H} \beta$. A range of nuclear mass and accretion rate across the Seyfert population can explain the differences observed in X-ray and optical properties. An attractive alternative model, which does not depend on any systematic difference in central mass, is that the circumnuclear gas of NLSy1s is different to BLSy1s in temperature, optical depth, density or geometry.
\end{abstract}

Subject headings: galaxies:active - galaxies:nuclei - X-rays: galaxies

\section{Introduction}

X-ray flux variability has long been known to be a common property of active galactic nuclei (AGN). Ariel V and HEAO-1 first revealed long term (days to years) variability in AGN (e.g. Marshall, Warwick \& Pounds 1981). It was not until the long-duration and uninterrupted observations of EXOSAT that rapid (thousands of seconds) variability was also established as common in these sources. X-ray flux variations are observed on

\footnotetext{
${ }^{1}$ Laboratory for High Energy Astrophysics, Code 660, NASA/Goddard Space Flight Center, Greenbelt, MD 20771

${ }^{2}$ University of Maryland Baltimore County, 1000 Hilltop Circle, Baltimore, MD 21250

${ }^{3}$ Universities Space Research Association

${ }^{4}$ University of Maryland College Park
} 
timescales from $\sim$ a thousand seconds to years and amplitude variations of up to an order of magnitude are observed in the $\sim 0.1-10 \mathrm{keV}$ band (also see review by Turner 1992 and references therein). Barr \& Mushotzky (1986) suggested the flux-doubling timescale of an AGN to be inversely proportional to its luminosity, both properties measured in the hard X-ray regime. The use of "doubling timescale" was a somewhat unsatisfactory quantification of the X-ray variability as, for many sources, this had to be derived by extrapolation of lower amplitude events. Lawrence \& Papadakis (1993) confirmed the variability-luminosity relationship, quantifying variations seen in EXOSAT light curves by using the timescale above which the integrated rms variation should be $10 \%$. Green, McHardy and Lehto (1993) also confirmed the correlation using the "normalized variability amplitude" and further, suggested sources with steeper X-ray spectra showed the highest amplitude of variability, perhaps due to a lower contribution from a reprocessed component. The existence of a correlation between X-ray variability and spectral slope was supported by the work of Koenig, Staubert \& Wilms (1997), using a different quantification of X-ray flux variability. Boller et al. (1996) examined the ROSAT "variation timescale" as a function of luminosity, concluding the high amplitude of variability observed in narrow-line Seyfert 1 galaxies to be inconsistent with scattering in an extended region, like that suggested for Seyfert 2 galaxies.

Nandra et al. 1997a (N97a) initiated use of "excess-variance" , $\sigma_{\text {RMS }}^{2}$ to quantify the X-ray variability of AGN (see below). This quantity had been previously used in analysis of the ultraviolet time series of AGN where Edelson, Krolik \& Pike (1990) found an anticorrelation between excess-variance and luminosity. Using excess-variance, N97a found an inverse correlation between X-ray variability (sampled using $128 \mathrm{~s}$ bins) and luminosity for a sample of Seyfert 1 galaxies with predominantly broad permitted lines. Fiore et al. (1998) used ROSAT HRI observations of a sample of quasars to examine correlations between the (soft) X-ray parameters of excess-variance, spectral index and luminosity, finding steep-spectrum, narrow-line quasars to show larger amplitude of X-ray variability in the $0.1-2 \mathrm{keV}$ band than the flat-spectrum broad-line quasars.

We recognize that there is a continuous distribution of optical line widths in Seyfert 1 galaxies, and any attempt to split these into narrow-line and broad-line Seyfert $1 \mathrm{~s}$ is arbitrary. However, for convenience we do make such a split here by referring to objects with FWHM H $\beta<2000 \mathrm{~km} / \mathrm{s}$ as narrow-line Seyfert 1s (NLSy1s) and objects with FWHM $\mathrm{H} \beta>2000 \mathrm{~km} / \mathrm{s}$ as broad-line Seyfert 1s (BLSy1s). This is purely to facilitate a concise discussion of the different regimes within the Seyfert 1 distribution.

The NLSy1 Ton S180 was found to lie significantly above the correlation established for the N97a sample (Turner et al. 1998) and also showed energy-dependent variability. 
The TON S180 result indicated that analysis of a sample including a significant number of NLSy1s may yield further insight into the physical processes at work in AGN.

This paper presents a timing analysis of 79 observations of 36 Seyfert 1 galaxies. Our sample consists of Seyfert 1 galaxies available from the Advanced Satellite for Cosmology and Astrophysics $(A S C A)$ archive up to November 1998. The purpose of this investigation was to expand upon the X-ray timing results presented in N97a, and to search for confirmation of some correlations between hard X-ray and optical parameters, suggested by previous works (above). In particular, we aimed to determine whether the established luminosity-variability correlation would alter when Seyfert galaxies covering the full observed range of permitted line widths were considered. The objects presented here do not form a complete sample. Nevertheless we suggest the results from this large collection of objects provides important new insight into the AGN phenomenon.

\section{ASCA Observations and Data Reduction}

ASCA has two solid-state imaging spectrometers (SISs; Burke et al. 1994) and two gas imaging spectrometers (GISs; Ohashi et al. 1996) yielding data over an effective bandpasses $\sim 0.5-10 \mathrm{keV}$. The data presented here were systematically reduced in the same way as the Seyfert galaxies presented in N97a, using source events within extraction cells of radii 4.8 and 6.6 arcmin for the SIS and GIS data, respectively.

In the timing analysis we used only data from the SIS instruments. We required that time series included in our analysis have at least 20 counts per time bin, and at least 20 bins in the final light curve. To increase the signal-to-noise ratio, we combined the SIS0 and SIS1 detectors requiring all time bins to be at least $99 \%$ exposed. The background level was not subtracted from these light curves. The excess-variance quantifies amplitude of variability in excess of that expected from statistical fluctuations in the background level. For each dataset we verified that the background light curve did not contain any significant trends. N97a use a 128s time bin for the light curve analysis. However, many of our AGN are faint, and analysis using a $128 \mathrm{~s}$ time bin would have lead to the exclusion of many potentially interesting sources. We found $256 \mathrm{~s}$ to be the optimum bin size for inclusion of most of the available data (use of longer integration times would have resulted in too few bins for many datasets).

As noted above, N97a introduced the use of the quantity normalized 'excess-variance,

$\sigma_{\text {RMS }}^{2}$ for analysis of $A S C A$ time series. Following that paper we designate the count rates for the $N$ points in each light curve as $X_{\mathrm{i}}$, with errors $\sigma_{\mathrm{i}}$. We further define $\mu$ as the 
unweighted, arithmetic mean of the $X_{\mathrm{i}}$. Then:

$$
\sigma_{\mathrm{RMS}}^{2}=\frac{1}{N \mu^{2}} \sum_{i=1}^{N}\left[\left(X_{\mathrm{i}}-\mu\right)^{2}-\sigma_{\mathrm{i}}^{2}\right]
$$

The error on $\sigma_{\mathrm{RMS}}^{2}$, is given by $s_{\mathrm{D}} /\left(\mu^{2} \sqrt{N}\right)$ where:

$$
s_{\mathrm{D}}^{2}=\frac{1}{N-1} \sum_{i=1}^{N}\left\{\left[\left(X_{i}-\mu\right)^{2}-\sigma_{i}^{2}\right]-\sigma_{\mathrm{RMS}}^{2} \mu^{2}\right\}^{2}
$$

i.e. the variance of the quantity $\left(X_{i}-\mu\right)^{2}-\sigma_{i}^{2}$. (Note there was a typographical error in N97a in that the equation for the error on $\sigma_{\mathrm{RMS}}^{2}$ should have had the quantity inside the summation squared, as shown here). We refer the reader to N97a for a discussion of the merits of using this quantity for timing analysis.

We note that for perfect comparison between sources, the excess-variance should be calculated using observations of the same duration $\left(T_{D}\right)$. However, in practice this would limit the analysis by reducing the number of sequences we could consider, or would require truncation of light curves to match the shortest observation. We note many of our light curves were constructed from observations of duration close to $50 \mathrm{ks}$. Our analysis includes observations both a factor of 2 smaller and larger than this. As the duration of an observation is random with respect to the parameters of interest then the effect of allowing a range of observation durations is to introduce scatter, which could hide some weak correlations but which should not introduce false correlations (because $T_{D}$ is not correlated with $\Gamma$, luminosity or FWHM $\mathrm{H} \beta$ ).

\section{Results}

We recalculated $\sigma_{\mathrm{RMS}}^{2}$ for the N97a sample of objects and all the new data presented here using $256 \mathrm{~s}$ time bins. Table 1 shows the datasets used in our analysis. The following sequences were also analyzed, but failed to meet our criteria for counts per bin and/or number of bins in the final light curve: Fairall-9 (73011050); NGC 4151 (70000000, 70000010, 71019030, 71019000); NGC 6814 (70012000); Mrk 509 (74024000, 74024010, 74024020); NGC 7469 (71028000, 71028030, 71028010); MCG -2-28-22 (70004000); ESO 141-G55 (72026000); PG1211+143 (70025000); Mrk 957(75057000) and Mrk 507(74033000).

The results are shown in Figure 1a. The dark squares denote NLSy1s, BLSy1s are denoted by a simple cross. Error bars are $1 \sigma$. Compared to the N97a sample, we have most 
notably added a significant number of objects with relatively narrow permitted lines and a number of points at the high luminosity end of the distribution (with multiple observations of Mrk 509 and Fairall-9).

Examination of Figure 1a shows that there remains a significant anticorrelation between $\sigma_{\text {RMS }}^{2}$ and luminosity (a Spearman-Rank correlation coefficient of -0.683 is obtained, significant at $>99 \%$ confidence). However there is a much greater degree of scatter in the correlation than in that shown by N97a. It is evident that this is predominantly due to the inclusion of a relatively large number of NLSy1 objects. This was expected, based upon the Ton S180 result (Turner et al. 1998) and was suggested by the ROSAT results of Boller et al. (1996). This result has also been found in a large independent study of NLSy1s (Leighly et al. 1999). This scatter suggests that $\sigma_{\text {RMS }}^{2}$ has a strong dependence on FWHM $\mathrm{H} \beta$ in addition to the dependence on luminosity. This does not weaken the dependence of $\sigma_{\text {RMS }}^{2}$ on luminosity, in the sense that it appears that objects with similar values of FWHM $\mathrm{H} \beta$ still show a correlation between $\sigma_{\text {RMS }}^{2}$ and luminosity.

NLSy1s are known to be steep, even in the ASCA bandpass (Brandt et al. 1997), and ROSAT data showed them to be generally highly variable X-ray sources (e.g. Boller et al. 1996, 1997; Forster \& Halpern 1996). Work by Green et al. (1993) showed a relationship between X-ray index and "normalized variability amplitude" in a sample of EXOSAT observations of AGN. The Green et al (1993) result was supported by the results of Koenig et al (1997). Hence we examined the relationship between $\sigma_{\text {RMS }}^{2}$ and the $2-10 \mathrm{keV}$ photon index $\Gamma_{2-10}$. The photon indices ( Table 1) were determined in the (rest-frame) 2-10 $\mathrm{keV}$ band for each source, excluding the (rest-frame) $5-7.5 \mathrm{keV}$ band which can contain significant photons from an Fe K $\alpha$ line (confusing measurements of the hard X-ray index). The photon index was derived fitting the two SIS and GIS datasets simultaneously, and using background spectra extracted from source-free regions of the detectors. A small difference in the normalization of each instrument was allowed, to account for slight differences in the flux calibration of each $(<20 \%)$. Absorption was included in the spectral model, but the column densities were allowed to be relatively unconstrained (simply greater than or equal to the Galactic line-of-sight value in each case). This resulted in conservative error bars for $\Gamma$. Column densities were generally consistent with Galactic values and are not tabulated here. Some values of $\Gamma_{2-10}$ were taken from N97b and George et al. (1998a), as noted in Table 1. These quantities are plotted in Figure 1b, and the correlation yielded a Spearman Rank coefficient of 0.412 . While the correlation is significant at $>99 \%$ confidence, the figure could be interpreted as showing that NLSy1 and BLSy1 objects occupy different areas of parameter space, with some zones-of-avoidance. Sources with steep photon indices, $\Gamma_{2-10}$ show relatively large amplitudes of variability when sampled on a timescale of $256 \mathrm{~s}$, compared to flat-spectrum objects. 
Next we examined directly the relationship between FWHM $\mathrm{H} \beta$ and $\sigma_{\mathrm{RMS}}^{2}$. This has not previously been done in a quantitative manner using $A S C A$ data. Values of FWHM $\mathrm{H} \beta$ were extracted from the literature and are shown in Table 1 . We caution the reader that many sources are known to show significant variations in FWHM H $\beta$ over timescales of years, and we take just one representative value for each source. In particular, Mrk 290 has reported values for FWHM $\mathrm{H} \beta$ which vary a great deal, we have used the Osterbrock (1977) value but note a much wider line of FWHM H $\beta=5340 \mathrm{~km} / \mathrm{s}$ has also been reported (Boroson \& Green, 1992). (We also caution that the least reliable numbers in Table 1 are the FWHM H $\beta$ for EXO 055620-3 and Nab 0205+024, which we measured from published optical spectra, but we deemed these estimations adequate for the purpose of this paper).

Figure 1c shows a very strong correlation between FWHM $\mathrm{H} \beta$ and $\sigma_{\mathrm{RMS}}^{2}$. The Spearman-Rank correlation coefficient was -0.723 , significant at $>99 \%$ confidence, and we find $\sigma_{\mathrm{RMS}}^{2} \propto(\mathrm{FWHM} \mathrm{H} \beta)^{-2.8}$. It is remarkable that this correlation should appear so strong given the line width variability noted above, and the lack of simultaneity of the optical and X-ray measurements. There must be a very strong underlying link between these two observables, which we will discuss in detail later.

As there is a known correlation between $\mathrm{EW} \mathrm{H} \beta$ and $\mathrm{FWHM} \mathrm{H} \beta$ (Boroson \& Green 1992), one would expect to also see a correlation of $\sigma_{\mathrm{RMS}}^{2}$ with $\mathrm{EW} \mathrm{H} \beta$. Indeed these quantities appear linked (Figure 1d) with a Spearman Rank correlation coefficient of -0.523 (significant at $>99 \%$ confidence). However, as we have relatively few measurements available for $\mathrm{EW} \mathrm{H} \beta$, we concentrate on the correlation between excess-variance and FWHM $\mathrm{H} \beta$.

It is also evident that significant changes are observed in $\sigma_{\mathrm{RMS}}^{2}$ between X-ray observations of a single source, although only Fairall-9, NGC 3783, NGC 5548 and Mrk 509 were observed frequently enough to look for this. In fact N97a previously noted changes in $\sigma_{\mathrm{RMS}}^{2}$ for NGC 4151, and George et al. 1998(b) noted them for NGC 3783. As the observations of any particular source are generally of approximately the same duration, these results indicate that the process producing the variability is non-stationary (Table 1).

\section{Discussion}

Assuming the bulk motion of the broad-line-gas is virialized, then differences in line width can be attributed to the emitting gas existing further from the nuclei of NLSy1s than

BLSy1s; alternatively the gas is at the same radius in both but the mass of the central black hole is smaller in NLSy1s than in BLSy1s. Our strongest correlation appears between $\sigma_{\text {RMS }}^{2}$ 
and FWHM $\mathrm{H} \beta$, and is consistent with rapid variability and narrow lines being a result of small central mass. The X-ray luminosities show comparable distributions for NLSy1s and BLSy1s. Taking a BLSy1 and NLSy1 of the same X-ray luminosity, then the BLSy1 will be a relatively high-mass system and the NLSy1 will be a low-mass system radiating at a high fraction of its Eddington luminosity to achive the same luminosity (yet having more rapid variability for that luminosity). These simple arguments have been discussed by numerous authors (e.g. Laor et al 1997). When both extremes of the Seyfert 1 phenomenon are considered in a variability-luminosity analysis then it includes sources with a range of accretion rates producing the same luminosity, this naturally introduces significant scatter into the correlation.

We confirm the findings of Green et al (1993) and Koenig et al (1997), that Seyferts with flat X-ray spectra (in the $2-10 \mathrm{keV}$ band) show relatively low amplitude of X-ray variability (when sampled on 256s) compared to the steep sources. The relatively high accretion rate inferred for NLSy1s may result in different conditions in the accretion disk and its corona. A higher optical depth of the corona in BLSy1s (compared to NLSy1s) would produce the harder X-ray spectrum observed. Another key parameter is coronal temperature, systematically cooler coronae could exist in NLSy1s, producing few hard $\mathrm{X}$-ray photons. The size of the Comptonization region is also important, an extended region will smear the intrinsic nuclear variability. Similarly, the geometry of the Comptonizing gas is important, relative to our line-of-sight.

A serious concern for models based upon a fundamental difference in central mass is that Rodriguez-Pascual et al. (1997) find broad components to emission lines observed in the UV band of both NLSy1s and BLSy1s. Again, assuming this gas is virialized it then contradicts the hypothesis of systematically smaller masses in NLSy1s than BLSy1s. We consider whether there is a model to explain the observed effects without relying on a relatively small central mass for NLSy1s. One clue may come from the observation of energy-dependent variability in TON S180 (Turner et al. 1998) and some BLSy1s (N97a, George et al. 1998a,c). It appears that in those sources the hard X-rays have traversed a longer path than soft X-ray photons, probably due to Compton-upscattering of some fraction of the nuclear continuum. Thus differences in the coronal conditions alone could explain both the difference in X-ray variability and spectral properties between BLSy1s and NLSy1s, with no inference on central mass. The problem with this picture is that the origin of the coronal differences is unknown, and our data require differences in coronal conditions to lead to differences in observed FWHM H $\beta$. Rodriguez-Pascual et al. (1997) have suggested that the line-emitting material is optically thin in NLSy1s, and that observed properties of any Seyfert depend on the gradient of density and ionization-state of the circumnuclear material around the Seyfert nuclei. The extension of this to our results 
indicates differences in nuclear environment, rather than central mass, can explain the range of observational properties of each type of Seyfert galaxy.

Some previous works have explained the narrow widths of optical lines in NLSyls by obscuration of the inner region of high-velocity gas evident in BLSy1s. This model now

seems unlikely, since X-ray observations have revealed little or no absorption of the nuclei of NLSy1s (e.g. Boller et al. 1996, Comastri et al. 1998, Turner et al. 1998).

\section{Conclusions}

The correlation established previously between X-ray variability and nuclear luminosity contains significant scatter when NLSy1 galaxies are included in the analysis, this result is in agreement with that found in the independent study of Leighly et al (1999). A strong correlation is also found between variability and $\mathrm{FWHM} \mathrm{H} \beta$. A range of nuclear mass and accretion rate can explain differences in X-ray variability, X-ray spectrum and optical-line-widths across the Seyfert population. However, evidence from UV data suggests we must consider models which do not hinge on differences in central mass. An alternative model is that the environment of the nuclei of NLSy1s is significantly different to BLSy1s. Differences in temperature, optical depth, density and geometry of circumnuclear gas can explain all observational results.

We are grateful to the $A S C A$ team for their operation of the satellite. This research has made use of the NASA/IPAC Extragalactic database, which is operated by the Jet Propulsion Laboratory, Caltech, under contract with NASA; of the Simbad database, operated at CDS, Strasbourg, France; and data obtained through the HEASARC on-line service, provided by NASA/GSFC. This work was supported by NASA Long Term Space Astrophysics grant NAG 5-7385.

\section{REFERENCES}

Barr, P., Mushotzky, R.F. 1986, Nature 320, 421

Boller, T., Brandt, W.N., Fink, H.H., 1996, A\&A, 305, 53

Boller, T., Brandt, W.N., Fabian, A.C. \& Fink, H.H., 1997, MNRAS, 289, 393 

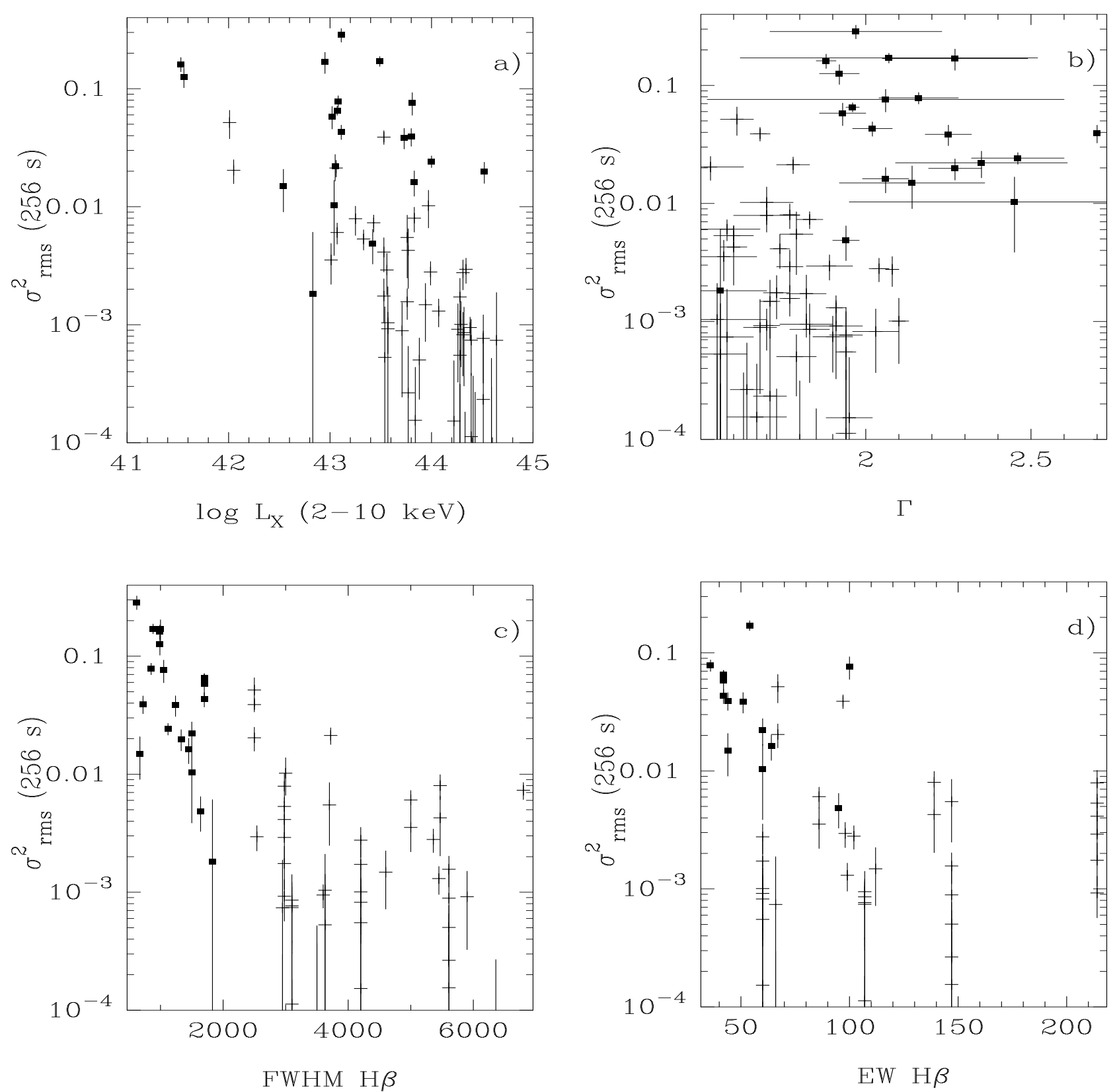

Fig. 1.- The excess-variance $\sigma_{r m s}^{2}$ in the $0.5-10 \mathrm{keV}$ band, from the combined SIS data, versus a) the rest-frame $2-10 \mathrm{keV}$ luminosity in erg/s. b) $\Gamma_{2-10}$ c) $\mathrm{FWHM} \mathrm{H} \beta$ in $\mathrm{km} / \mathrm{s} \mathrm{d}$ ) EW $\mathrm{H} \beta$ in $\AA$. Sources with FWHM $\mathrm{H} \beta<2000 \mathrm{~km} / \mathrm{s}$ are shown as black squares, the rest of the sample are open crosses and error bars are $1 \sigma$. 
Boroson, T.A., Green, R.F. 1992, ApJS 80, 109

Brandt, W.N., Mather, S., Elvis, M., 1997, MNRAS, 285, L25

Burke, B.E., Mountain, R.W., Daniels, P.J., Dolat, V.S., 1994, IEEE Trans. Nuc. SCI. 41, 375

Comastri, A. et al. 1998, A\&A, 333, 31

Crenshaw, D.M. 1986, ApJS 62, 821

Cruz-Gonzalez, I., Carrasco, L., Serrano, A. Guichard, J., Dultzin-Hacyan, D., Bisiacchi, G. F. 1994, ApJS 94, 47

Dietrich, M et al. 1998, ApJS 115, 185

Edelson, R., Krolik, J.H., Pike, G.F. 1990, ApJ 359, 86

Fairall, A. P., Mchardy, I. M., Pye, J. P. 1982, MNRAS 198, 13

Fiore, F., Laor, A., Elvis, M., Nicastro, F., Giallongo, E., 1998, ApJ 503, 607

Forster, K., Halpern, J.P., 1996, ApJ, 468, 565

George, I.M., Turner, T.J., Netzer, H., Nandra, K., Mushotzky, R.F., Yaqoob.T., 1998a, ApJS 114, 73

George, I.M., Turner, T.J., Mushotzky, R.F., Nandra, K., Netzer, H. 1998b, ApJ, 503, 174

George, I.M., Mushotzky, R.F., Turner, T.J., Yaqoob, Y., Ptak, A., Nandra, K., Netzer, H., 1998c, ApJ, 509, 146

George, I.M., et al. 1999, in prep.

Goodrich, R.W. 1989, ApJ 342, 224

Green, A. McHardy, I.M., Lehto, H.J. 1993, MNRAS 265, 664

Grupe, D., 1996, PhD Thesis, Gottingen University, F.R.G.

Koenig, M., Staubert, R., Wilms, J. 1997, A\&A 326, L25

Laor, A., Fiore, F., Elvis, M., Wilkes, B.J., McDowell, J.C. 1997, ApJ 477, 93

Lawrence, A., Papadakis, I. 1993, ApJ 414, L85

Leighly, K., et al. 1999, ApJ submitted.

Marshall, F.E., Warwick, R.S., Pounds, K.A. 1981, MNRAS 194, 987

Marziani, P., Sulentic, J.W., Dultzin-Hacyan, D., Calvani, M., Moles, M. 1996, ApJS 104, 37

Morris, S.L., Ward, M.J. 1988, MNRAS 230, 639 
Nandra, K., George, I.M., Mushotzky, R.F., Turner, T.J., Yaqoob, T., 1997, ApJ, 476, 70 (N97a)

Nandra, K., George, I.M., Mushotzky, R.F., Turner, T.J., Yaqoob, T., 1997, ApJ, 477, 602 (N97b)

Ohashi, T., et al., 1996, PASJ, 48, 157

Osterbrock, D.E., 1977, ApJ 215, 733

Osterbrock, D.E., Shuder, J.M. 1982, ApJS 49, 149

Pineda, F. J., Delvaille, J. P., Schnopper, H. W., Grindlay, J. E. 1980, ApJ 237, 414

Puchnarewicz, E.M., Mason, K.O., Siemiginowska, A., Pounds, K.A. 1995, MNRAS, 276, 20

Rodriguez-Pascual, P.M, Mas-Hesse, J.M., Santos-Lleo, M. 1997, A\&A, 327, 72

Schmidt, G.D., Miller, J.S. 1980, ApJ240, 759

Stirpe, G. 1990, A\&AS 85, 1049

Turner, T.J. 1992, in "The Nature of Compact Objects in Active Galactic Nuclei" Ed. Andrew Robinson \& Roberto Terlevich, Cambridge University Press

Turner, T.J., George, I.M., Nandra, K. 1998, ApJ 508, 648

Wilson, A.S., Penston, M.V. 1979, ApJ232, 389 
Table 1. Optical and X-ray Parameters for Seyfert Galaxies

\begin{tabular}{|c|c|c|c|c|c|c|c|}
\hline Source & Sequence & $10^{-3} \sigma_{r m s(256)}^{2}$ & $\log L_{2-10}$ & $\Gamma$ & $\begin{array}{c}\mathrm{T}_{D} / \mathrm{T}_{E} \\
\mathrm{ks}\end{array}$ & $\begin{array}{c}\text { FWHM H } \beta^{5} \\
\mathrm{~km} / \mathrm{s}\end{array}$ & $\begin{array}{c}\mathrm{EW} \mathrm{H} \beta^{5} \\
\AA\end{array}$ \\
\hline Mrk 335 & 71010000 & $4.86_{-}^{+} 1.59$ & 43.42 & $1.94_{-}^{+} 0.04^{1}$ & $46.8 / 18.9$ & $1640^{6}$ & $95^{6}$ \\
\hline $1 Z W$ I & 73042000 & $38.5_{-}^{+} 7.63$ & 43.73 & $2.25_{-}^{+} 0.07^{2}$ & $92.3 / 27.1$ & $1240^{6}$ & $51^{6}$ \\
\hline TON S180 & 74081000 & $24.2_{-}^{+} 2.75$ & 44.0 & $2.46_{-}^{+} 0.14$ & $50.4 / 44.4$ & $1120^{7}$ & $\ldots$ \\
\hline Fairall-9 & 71027000 & $0.92_{-}^{+} 0.59$ & 44.26 & $1.91_{-}^{+} 0.08^{1}$ & $53.3 / 20.8$ & 5900 & 60 \\
\hline$\cdots$ & 73011000 & $0.82_{-}^{+} 0.45$ & 44.31 & $2.03_{-}^{+} 0.07$ & $57.8 / 16.2$ & $\cdots$ & $\cdots$ \\
\hline$\cdots$ & 73011010 & $0.15_{-}^{+} 0.34$ & 44.22 & $1.95_{-}^{+} 0.07$ & $55.4 / 14.2$ & $\cdots$ & $\cdots$ \\
\hline$\ldots$ & 73011020 & $1.72_{-}^{+} 0.75$ & 44.28 & $1.82_{-}^{+} 0.06$ & $45.7 / 16.6$ & $\cdots$ & $\ldots$ \\
\hline$\ldots$ & 73011030 & $0.55_{-}^{+} 0.77$ & 44.28 & $1.94_{-}^{+} 0.03$ & $38.2 / 15.5$ & $\ldots$ & $\ldots$ \\
\hline$\ldots$ & 73011040 & $2.76_{-}^{+} 0.79$ & 44.31 & $2.08_{-}^{+} 0.02$ & $38.3 / 21.0$ & $\cdots$ & $\ldots$ \\
\hline$\cdots$ & 73011060 & $1.01_{-}^{+} 0.57$ & 44.29 & $2.10_{-}^{+} 0.03$ & $25.0 / 16.3$ & $\ldots$ & $\ldots$ \\
\hline RX J 0148-27 & 75043000 & $76.1_{-}^{+} 16.5$ & 43.81 & $2.06_{-}^{+} 0.54$ & $106 / 34.4$ & $1050^{8}$ & $100^{8}$ \\
\hline Nab $0205+024$ & 74071000 & $19.8_{-}^{+} 4.03$ & 44.52 & $2.27_{-}^{+} 0.08$ & $118 / 48.8$ & $1330^{9}$ & $\cdots$ \\
\hline Mrk 1040 & 72016000 & $1.82_{-}^{+} 4.26$ & 42.83 & $1.56_{-}^{+} 0.14$ & $41.5 / 14.1$ & $1830^{10}$ & $\ldots$ \\
\hline 3C 111 & 74087000 & $0.23_{-}^{+} 0.65$ & 44.51 & $1.71_{-}^{+} 0.05$ & $81.5 / 32.5$ & $\ldots$ & $\ldots$ \\
\hline LB 1727 & 74056000 & $0.00_{-}^{+} 1.21$ & 44.59 & $1.56_{-}^{+} 0.14$ & $77.4 / 23.5$ & $2800^{8}$ & $66^{8}$ \\
\hline$\ldots$ & 74056010 & $0.74_{-}^{+} 1.14$ & 44.64 & $1.58_{-}^{+} 0.08$ & $71.6 / 23.2$ & $\ldots$ & $\cdots$ \\
\hline $3 \mathrm{C} 120$ & 71014000 & $2.95_{-}^{+} 0.71$ & 44.34 & $1.89_{-}^{+} 0.07^{1}$ & $128 / 46.0$ & 2540 & 98 \\
\hline Ark 120 & 72000000 & $1.31_{-}^{+} 0.35$ & 44.07 & $1.91_{-}^{+} 0.03$ & $99.9 / 36.1$ & 5450 & 99 \\
\hline MCG 8-11-11 & 73052000 & $0.53_{-}^{+} 0.89$ & 43.54 & $1.56_{-}^{+} 0.08$ & $25.2 / 11.3$ & $3630^{10}$ & $\ldots$ \\
\hline$\ldots$ & 73052010 & $1.04_{-}^{+} 1.06$ & 43.57 & $1.55_{-}^{+} 0.15$ & $25.5 / 11.1$ & $\ldots$ & $\ldots$ \\
\hline H0707-495 & 73043000 & $169_{-}^{+} 34.5$ & 42.95 & $2.27_{-}^{+} 0.22$ & $92.6 / 33.9$ & $1000^{11}$ & $\ldots$ \\
\hline NGC 3227 & 70013000 & $51.7_{-}^{+} 14.0$ & 42.01 & $1.61_{-}^{+} 0.05^{3}$ & $84.2 / 32.7$ & $2500^{12}$ & 67 \\
\hline$\ldots$ & 73068000 & $20.34_{-}^{+} 4.7$ & 42.05 & $1.53_{-}^{+} 0.10^{3}$ & $83.4 / 34.3$ & $\ldots$ & $\cdots$ \\
\hline RE $1034+396$ & 72020000 & $22.1_{-}^{+} 5.63$ & 43.05 & $2.35_{-}^{+} 0.26$ & $82.6 / 28.5$ & $1500^{13}$ & 60 \\
\hline$\ldots$ & 72020010 & $10.3_{-}^{+} 6.44$ & 43.04 & $2.45_{-}^{+} 0.50$ & $22.4 / 11.0$ & $\ldots$ & $\ldots$ \\
\hline NGC 3516 & 71007000 & $7.31_{-}^{+} 1.21$ & 43.43 & $1.83_{-}^{+} 0.04$ & $77.5 / 28.8$ & $6800^{12}$ & $\ldots$ \\
\hline NGC 3783 & 71041000 & $7.91_{-}^{+} 2.20$ & 43.25 & $1.70_{-}^{+} 0.10^{4}$ & $36.7 / 17.2$ & 2980 & 214 \\
\hline$\ldots$ & 71041010 & $5.33_{-}^{+} 1.04$ & 43.33 & $1.60_{-}^{+} 0.06^{4}$ & $31.7 / 15.1$ & $\ldots$ & $\ldots$ \\
\hline$\cdots$ & 74054000 & $0.92_{-}^{+} 0.36$ & 43.57 & $1.70_{-}^{+} 0.04^{4}$ & $37.6 / 14.9$ & $\ldots$ & $\ldots$ \\
\hline$\ldots$ & 74054010 & $1.75_{-}^{+} 0.70$ & 43.53 & $1.73_{-}^{+} 0.04^{4}$ & $54.8 / 18.7$ & $\cdots$ & $\ldots$ \\
\hline$\cdots$ & 74054020 & $2.91_{-}^{+} 1.12$ & 43.56 & $1.77_{-}^{+} 0.04^{4}$ & $59.2 / 15.5$ & $\cdots$ & $\cdots$ \\
\hline$\ldots$ & 74054030 & $4.13_{-}^{+} 1.33$ & 43.53 & $1.74_{-}^{+} 0.04^{4}$ & $57.5 / 14.8$ & $\ldots$ & $\ldots$ \\
\hline Mrk 42 & 75056000 & $14.9_{-}^{+} 5.87$ & 42.54 & $2.14_{-}^{+} 0.22$ & $81.0 / 36.4$ & $670^{14}$ & 44 \\
\hline \multirow[t]{2}{*}{ NGC 4051} & 70001000 & $126_{-}^{+} 24.0$ & 41.56 & $1.92_{-}^{+} 0.06^{1}$ & $82.1 / 26.0$ & $990^{10}$ & $\ldots$ \\
\hline & 72001000 & $162_{-}^{+} 22.4$ & 41.53 & $1.88_{-}^{+} 0.03$ & $159 / 70.1$ & $\ldots$ & $\cdots$ \\
\hline NGC 4151 & 71019020 & $3.54_{-}^{+} 1.34$ & 43.01 & $1.57_{-}^{+} 0.10^{1}$ & $25.5 / 10.9$ & $5000^{15}$ & 86 \\
\hline$\ldots$ & 71019010 & $6.05_{-}^{+} 1.23$ & 43.07 & $1.58_{-}^{+} 0.10^{1}$ & $25.0 / 12.2$ & $\ldots$ & $\ldots$ \\
\hline Mrk 766 & 71046000 & $78.4_{-}^{+} 8.80$ & 43.08 & $2.16_{-}^{+} 0.12^{1}$ & $77.3 / 33.0$ & $850^{11}$ & $36^{16}$ \\
\hline
\end{tabular}


Table 1 - Continued

\begin{tabular}{|c|c|c|c|c|c|c|c|}
\hline Source & Sequence & $10^{-3} \sigma_{r m s(256)}^{2}$ & $\log L_{2-10}$ & $\Gamma$ & $\begin{array}{c}\mathrm{T}_{D} / \mathrm{T}_{E} \\
\mathrm{ks}\end{array}$ & $\begin{array}{c}\text { FWHM H } \beta^{5} \\
\mathrm{~km} / \mathrm{s}\end{array}$ & $\begin{array}{c}\mathrm{EW} \mathrm{H} \beta^{5} \\
\AA\end{array}$ \\
\hline NGC 4593 & 71024000 & $21.3_{-}^{+} 3.45$ & 43.06 & $1.78_{-}^{+} 0.05^{1}$ & $93.5 / 21.9$ & $3720^{17}$ & $\cdots$ \\
\hline IRAS 13224-3809 & 72011000 & $286_{-}^{+} 37.9$ & 43.11 & $1.97_{-}^{+} 0.26$ & $154 / 82.8$ & $620^{11}$ & $\ldots$ \\
\hline MCG -6-30-15 & 70016000 & $43.1_{-}^{+} 6.02$ & 43.11 & $2.02_{-}^{+} 0.06^{1}$ & $86.5 / 30.5$ & $1700^{18}$ & 42 \\
\hline$\ldots$ & 70016010 & $58.2_{-}^{+} 12.6$ & 43.02 & $1.93_{-}^{+} 0.07^{1}$ & $92.3 / 30.0$ & $\ldots$ & $\ldots$ \\
\hline$\ldots$ & 72013000 & $65.4_{-}^{+} 5.82$ & 43.07 & $1.96_{-}^{+} 0.14$ & $363 / 152$ & $\ldots$ & $\ldots$ \\
\hline EXO 055620-3 & 73070000 & $10.2_{-}^{+} 3.59$ & 43.97 & $1.70_{-}^{+} 0.08$ & $92.6 / 37.3$ & $3000^{19}$ & $112^{20}$ \\
\hline IC4329A & 70005000 & $1.48_{-}^{+} 0.76$ & 43.06 & $1.71_{-}^{+} 0.03^{1}$ & $82.1 / 28.1$ & $4800^{21}$ & $\ldots$ \\
\hline Mrk 279 & 72028000 & $2.80^{+} 0.63$ & 43.99 & $2.04_{-}^{+} 0.03$ & $42.2 / 21.3$ & 5360 & 102 \\
\hline PG $1404+226$ & 72021000 & $171_{-}^{+} 16.3$ & 43.49 & $2.07_{-}^{+} 0.45^{2}$ & $96.9 / 34.4$ & $880^{6}$ & $54^{6}$ \\
\hline NGC 5548 & 70018000 & $5.49_{-}^{+} 3.00$ & 43.76 & $1.79_{-}^{+} 0.05^{1}$ & $81.6 / 20.4$ & 5610 & 147 \\
\hline$\cdots$ & 74038000 & $0.50_{-}^{+} 0.27$ & 43.88 & $1.79_{-}^{+} 0.06$ & $57.1 / 17.4$ & $\ldots$ & $\ldots$ \\
\hline$\cdots$ & 74038010 & $1.57_{-}^{+} 0.46$ & 43.84 & $1.77_{-0.03}^{+}$ & $35.0 / 14.5$ & $\cdots$ & $\cdots$ \\
\hline$\cdots$ & 74038020 & $0.89_{-}^{+} 0.65$ & 43.76 & $1.68_{-}^{+} 0.05$ & $46.6 / 16.3$ & $\cdots$ & $\cdots$ \\
\hline$\cdots$ & 74038030 & $0.26_{-}^{+} 0.39$ & 43.71 & $1.64_{-}^{+} 0.05$ & $38.0 / 16.2$ & $\cdots$ & $\cdots$ \\
\hline$\cdots$ & 74038040 & $0.15_{-}^{+} 0.28$ & 43.77 & $1.67_{-}^{+} 0.09$ & $47.0 / 18.6$ & $\cdots$ & $\ldots$ \\
\hline Mrk 478 & 73067000 & $16.2_{-}^{+} 3.92$ & 43.83 & $2.06_{-}^{+} 0.07^{2}$ & $84.6 / 31.9$ & $1450^{6}$ & $64^{6}$ \\
\hline Mrk 841 & 70009000 & $8.00_{-}^{+} 1.90$ & 43.83 & $1.77_{-}^{+} 0.03^{2}$ & $78.2 / 30.3$ & $5470^{6}$ & $64^{6}$ \\
\hline$\ldots$ & 71040000 & $4.27_{-}^{+} 2.24$ & 43.02 & $1.60_{-}^{+} 0.04^{2}$ & $59.6 / 20.6$ & $\ldots$ & $\ldots$ \\
\hline Mrk 290 & 72027000 & $38.9_{-}^{+} 5.21$ & 43.53 & $1.68_{-}^{+} 0.03^{2}$ & $98.3 / 41.0$ & $2500^{12}$ & $87^{6}$ \\
\hline 3C 390.3 & 73082000 & $0.0_{-}^{+0.86}$ & 44.41 & $1.63_{-}^{+} 0.08$ & $37.8 / 16.8$ & 3500 & $\cdots$ \\
\hline$\ldots$ & 73082010 & $0.0_{-}^{+} 0.52$ & 44.59 & $1.55_{-}^{+} 0.15$ & $42.3 / 14.7$ & $\cdots$ & $\cdots$ \\
\hline Mrk 509 & 71013000 & $0.95_{-}^{+} 0.21$ & 44.38 & $1.82_{-}^{+} 0.08$ & $107 / 40.1$ & $3600^{12}$ & $107^{23}$ \\
\hline$\ldots$ & 74024030 & $0.77_{-}^{+} 0.45$ & 44.51 & $1.94_{-}^{+} 0.05$ & $25.6 / 11.5$ & $\ldots$ & $\ldots$ \\
\hline$\cdots$ & 74024040 & $0.11_{-}^{+} 0.28$ & 44.39 & $1.94_{-}^{+} 0.03$ & $23.3 / 8.42$ & $\cdots$ & $\cdots$ \\
\hline$\cdots$ & 74024050 & $0.00_{-}^{+} 0.12$ & 44.33 & $1.82_{-0.05}^{+}$ & $23.2 / 11.7$ & $\cdots$ & $\cdots$ \\
\hline$\cdots$ & 74024060 & $0.00_{-}^{+} 0.30$ & 44.28 & $1.80_{-}^{+} 0.06$ & $21.7 / 10.2$ & $\cdots$ & $\cdots$ \\
\hline$\cdots$ & 74024070 & $0.00_{-}^{+} 0.30$ & 44.33 & $1.85_{-}^{+} 0.11$ & $23.6 / 9.30$ & $\cdots$ & $\cdots$ \\
\hline$\cdots$ & 74024080 & $0.74_{-}^{+} 0.37$ & 44.39 & $1.90_{-}^{+} 0.06$ & $23.8 / 9.80$ & $\cdots$ & $\cdots$ \\
\hline$\cdots$ & 74024090 & $0.86_{-}^{+} 0.55$ & 44.32 & $1.83_{-}^{+} 0.06$ & $26.9 / 9.12$ & $\cdots$ & $\cdots$ \\
\hline Ark 564 & 74052000 & $39.3_{-}^{+2.24}$ & 43.80 & $2.70_{-}^{+} 0.02$ & $104 / 47.6$ & $720^{14}$ & $44^{14}$ \\
\hline MCG -2-58-22 & 75049010 & $0.00_{-}^{+} 0.21$ & 44.43 & $1.73_{-}^{+} 0.04$ & $99.1 / 36.7$ & $6360^{12}$ & $\cdots$ \\
\hline
\end{tabular}

Note. - Errors are $1 \sigma . \mathrm{T}_{D}$ is the duration and $\mathrm{T}_{E}$ the exposure of the observation. References: ${ }^{1}$ Nandra et al 1997b; ${ }^{2}$ George et al 1999; ${ }^{3}$ George et al 1998c ${ }^{4}$ George et al 1998b ${ }^{5}$ Stirpe 1990 unless otherwise noted ${ }^{6}$ Boroson \& Green $1992{ }^{7}$ Comastri et al $1998{ }^{8}$ Grupe $1996{ }^{9}$ Estimated from Gianuzzo \& Stirpe $1996{ }^{10}$ Osterbrock \& Shuder $1982{ }^{11}$ Boller, Brandt \& Fink $1996{ }^{12}$ Osterbrock $1977{ }^{13}$ Puchnarewicz et al $1995{ }^{14}$ Goodrich $1989{ }^{15}$ Schmidt \& Miller $1980{ }^{16}$ Cruz-Gonzales et al $1994{ }^{17}$ Crenshaw $1986{ }^{18}$ Pineda et al $1980{ }^{19}$ Estimated from Fairall, Mchardy \& Pye $1982{ }^{20}$ Morris \& Ward $1988{ }^{21}$ Wilson \& Penston $1979{ }^{22}$ Dietrich et al $1998{ }^{23}$ Marzioni et al 1996 